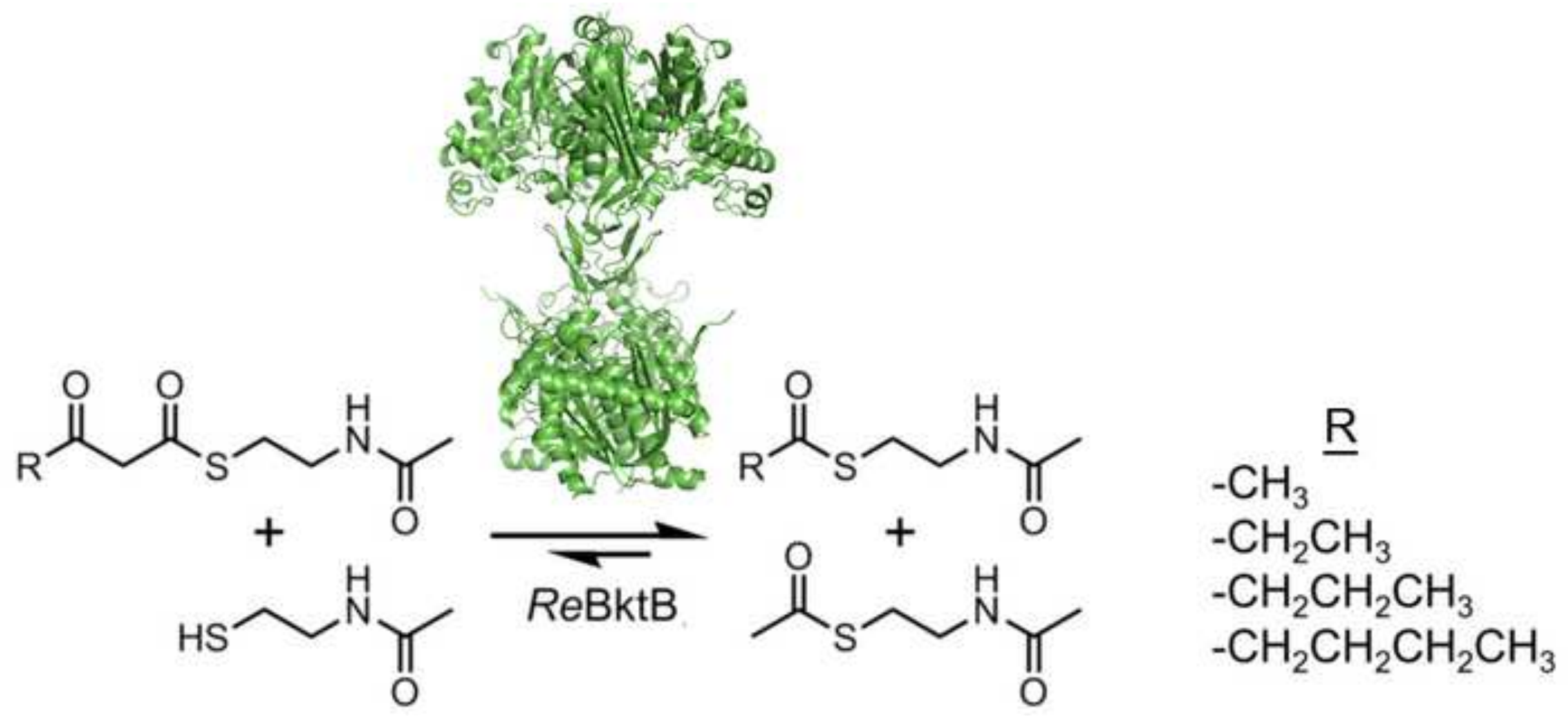




\title{
Coenzyme A-free activity, crystal structure, and rational engineering of a promiscuous $\beta$-ketoacyl thiolase from Ralstonia eutropha
}

\author{
Christopher D. Fage a , Jessica L. Meinke ${ }^{\text {a }}$, Adrian T. Keatinge-Clay ${ }^{\text {a,b,* }}$ \\ ${ }^{a}$ Department of Molecular Biosciences, The University of Texas, Austin, TX 78712, USA. \\ ${ }^{b}$ Department of Chemistry, The University of Texas, Austin, TX 78712, USA.
}

"Corresponding author: Tel.: (512) 471-2977, Email address: adriankc@utexas.edu 


\begin{abstract}
Thiolases catalyze the formation of carbon-carbon bonds in diverse biosynthetic pathways. The promiscuous $\beta$-ketoacyl thiolase $\mathrm{B}$ of Ralstonia eutropha (ReBktB) has been utilized in the in vivo conversion of Coenzyme A (CoA)-linked precursors such as acetyl-CoA and glycolyl-CoA into $\beta$ hydroxy acids, including the pharmaceutically-important 3,4-dihydroxybutyric acid. Such thiolases could serve as powerful carbon-carbon bond-forming biocatalysts in vitro if handles less costly than CoA were employable. Here, thiolase activity is demonstrated toward substrates linked to the readily-available CoA mimic, $N$-acetylcysteamine (NAC). ReBktB was observed to catalyze the retro-Claisen condensation of several $\beta$-ketoacyl-S-NAC substrates, with a preference for 3-oxopentanoyl-S-NAC over 3-oxobutanoyl-, 3-oxohexanoyl-, and 3-oxoheptanoyl-S-NAC. A $2.0 \AA$ A-resolution crystal structure, in which the asymmetric unit consists of four ReBktB tetramers, provides insight into acyl group specificity and how it may be engineered. By replacing an active site methionine with an alanine, a mutant possessing significant activity towards $\alpha$-methyl substituted, NAC-linked substrates was engineered. The ability of $R e \mathrm{BktB}$ and its engineered mutants to utilize NAC-linked substrates will facilitate the in vitro biocatalytic synthesis of diketide chiral building blocks from feedstock molecules such as acetate and propionate.
\end{abstract}




\section{Introduction}

Biosynthetic thiolases catalyze carbon-carbon bond formation in many pathways. The first step in the production of isoprenoids, ketone bodies, and polyhydroxyalkanoate (PHA) polymers is the thiolasemediated condensation of two molecules of acetyl-CoA into acetoacetyl-CoA [1,2]. Such carbon-carbon bond-forming reactions are of biocatalytic interest since they could enable the generation of complex organic compounds from feedstock chemicals.

The bacterium Ralstonia eutropha utilizes $\beta$-ketoacyl thiolases such as RePhbA (also known as $R e \mathrm{PhaA}$ ) and $R e \mathrm{BktB}$ in the biosynthesis of PHA polymers that store both carbon and energy (Figure 1a) [3-8]. While RePhbA is specific for acetyl-CoA and helps generate 3-hydroxybutyryl monomers in the biosynthesis of poly-(R)-3-hydroxybutanoate, $R e \mathrm{BktB}$ can condense acetyl-CoA with propionyl-CoA or butyryl-CoA to generate 3-hydroxypentanoyl or 3-hydroxyvaleroyl monomers in the biosynthesis of PHA copolymers. Recently, the promiscuous substrate specificity of $R e \mathrm{BktB}$ was harnessed by incorporating it within a pathway engineered into $E$. coli to yield chiral building blocks such as the pharmaceuticallyimportant 3,4-dihydroxybutyric acid (Figure $1 \mathrm{~b}$ ). In these studies, $R e$ BktB was observed to condense acetyl-CoA with acetyl-, propionyl-, butyryl-, isobutyryl-, and glycolyl-CoA [9,10]. Fascinatingly, the chiral building block 2,3-dihydroxybutyric acid was also generated by the engineered pathway, indicating that $R e \mathrm{BktB}$ produced the $\alpha$-substituted intermediate 2-hydroxy-3-oxobutryl-CoA from acetyl-CoA and glycolyl-CoA.

A biosynthetic thiolase fuses a priming acyl-CoA and an extending acyl-CoA into a $\beta$-ketoacylCoA through a Claisen-like condensation that proceeds through a two-step ping-pong mechanism $[1,11,12]$. Under standard conditions, the reverse reaction is thermodynamically favored; however, in biological contexts flux is usually in the forward direction owing to the removal of product by downstream enzymes in the biosynthetic pathway. Thus, for thiolases to be employed in the in vitro synthesis of chiral building blocks, they need to be coupled with energetically-favorable reactions (Figure 1c). Another obstacle in employing thiolases in vitro is the expense of the CoA handle. Binary complexes 
of CoA-bound biosynthetic thiolases, such as the thiolase from Zoogloea ramigera (referred to here as ZrPhbA, PDB 1DLV), show interactions with the CoA adenosine and pantetheinyl moieties that may accelerate catalysis [13]. To our knowledge, the importance of these interactions for thiolase-mediated catalysis has not been determined.

Here, we demonstrate the activity of ReBktB toward $\beta$-ketoacyl substrates linked to the readily available $N$-acetylcysteamine (NAC) handle that lacks the phosphoadenosine moiety and most of the pantetheinyl arm of CoA. In the degradative direction, $R e \mathrm{BktB}$ is shown to prefer 3-oxopentanoyl-S-NAC over 3-oxobutanoyl-S-NAC and is also active towards 3-oxohexanoyl-S-NAC and 3-oxoheptanoyl-SNAC. A 2.01- $\AA$ resolution crystal structure of $\operatorname{ReBktB}$ helps to provide a structural basis for the broad substrate specificity of the thiolase. Through rational engineering, a mutant was generated with significant activity towards $\alpha$-methyl substituted substrates.

\section{Results}

\subsection{In vitro thiolysis of $\beta$-ketoacyl-S-NAC substrates}

$\operatorname{ReBkB}(1 \mu \mathrm{M})$ was incubated with $5 \mathrm{mM}$ NAC and $1 \mathrm{mM} \beta$-ketoacyl-S-NAC substrate: 3oxobutanoyl-S-NAC (1), 3-oxopentanoyl-S-NAC (2), 3-oxohexanoyl-S-NAC (3), 3-oxoheptanoyl-S-NAC (4), or (2RS)-2-methyl-3-oxopentanoyl-S-NAC (5) (Figure 2). After $1 \mathrm{~h}$ and $24 \mathrm{~h}$ at $22{ }^{\circ} \mathrm{C}$, reactions were quenched with acid and analyzed by HPLC. The $\alpha$-branched $\mathbf{5}$ was found to be an unsuitable substrate for the enzyme even after a 24-h incubation (Figure S1). However, after $1 \mathrm{~h}$ reactions containing 1, 2, 3, or 4 yielded acetyl-S-NAC (6) as well as another molecule of $\mathbf{6}$, propionyl-S-NAC (7), butyryl-S-NAC (8), or valeryl-S-NAC (9), respectively (products showed the same retention times and absorbance spectra as synthetic standards, and their identities were corroborated by LC/MS: $m / z=162.2,176.2,190.2$, and 204.4 for 6-9); after $24 \mathrm{~h}$, the $\beta$-ketoacyl-S-NAC substrates had completely converted to acyl-S-NAC 
products. Peaks for product $\mathbf{6}$ were integrated and their areas were compared to a standard curve to determine the concentration of $\mathbf{6}$ formed (Figure S2). ReBktB exhibited preference for the $\beta$-ketoacyl-SNAC thioesters in the order $\mathbf{2}>\mathbf{1}>\mathbf{3} \sim \mathbf{4}(0.873 \pm 0.031 \mathrm{mM}, 0.238 \pm 0.007 \mathrm{mM}, 0.089 \pm 0.006 \mathrm{mM}$, and $0.082 \pm 0.005 \mathrm{mM} 6$ formed, respectively). This differs from the previously determined specificity of ReBktB toward $\beta$-ketoacyl-CoA thioesters (3-oxopentanoyl-CoA > 3-oxohexanoyl-CoA > 3oxobutanoyl-CoA) [5]. Increasing the concentration of $\operatorname{ReBktB}$ to $10 \mu \mathrm{M}$ resulted in the complete thiolysis of the 7-carbon NAC thioester 4 (the least favorable substrate tested) into 6 and 9 within $1 \mathrm{~h}$ (the chromatogram resembles that of the $24 \mathrm{~h}$ reaction with $1 \mu \mathrm{M} R e \mathrm{BktB}$; Figure S1). To our knowledge, utilization of $\beta$-ketoacyl-S-NAC substrates by a $\beta$-ketoacyl thiolase has not been reported.

\subsection{ReBktB crystallography}

$R e$ BktB crystallized in space group $P 1$ with 16 monomers per asymmetric unit (Figure 3a and Table 1). Molecular replacement was performed with Phaser by searching for eight copies of Clostridium difficile thiolase dimer (PDB 4DD5) [14]. Twinning was suspected after residual factors stalled during refinement despite the high quality of the electron density map [15]. The dataset was processed in a primitive triclinic unit cell and submitted to phenix.xtriage of the PHENIX software bundle [16], which estimated the twin fraction $\alpha>0.41$ (where $\alpha=0.5$ for perfectly twinned and $\alpha=0$ for untwinned) and identified three pseudo-merohedral twin operators: (1) $h,-k,-l$; (2) $-h, k,-l$; and (3) $-h,-k, l$ (Table S1). The software also performed an $L$ Test [17], calculating $\langle|L|\rangle=0.421$ and $\left\langle L^{2}\right\rangle=0.248$ for acentric reflections, which deviate significantly from expected values for normal, untwinned data (Figure S3). Twin refinement in REFMAC5 [18] of the CCP4 software suite [19] drastically improved the residual factors after a single cycle (Table S2). In the final model, $R_{\text {work }}=17.6 \%$ and $R_{\text {free }}=20.8 \%$. The only region between residues 2 and 393 that shows weak electron density is loop residues 207-210. The

presented crystal structure is higher quality (resolution: 2.01 vs. $2.29 \AA, B$-factors: 42.2 vs. $64.6 \AA^{2}$, 
$R_{\text {work }} / R_{\text {free: }}: 17.6 \% / 20.8 \%$ vs. $25.3 \% / 31.6 \%$, completeness: $92.9 \%$ vs. $85.9 \%$ ) than a recently-reported crystal structure of $\operatorname{ReBktB}[20]$.

The tertiary fold of $R e B k t B$ is highly similar to that of $Z r P h b A$, a biosynthetic thiolase from $Z$. ramigera (PDBs 1DLV and 1DM3, 51\% sequence identity) [13,21], with $\mathrm{C}_{\alpha}$ r.m.s.d. $=0.64 \AA$ over 339 residues (Figures $3 b$ and S4). The enzyme adopts the five-layered $\alpha \beta \alpha \beta \alpha$ fold of the thiolase superfamily in which two $\beta$-sheets are sandwiched between three clusters of $\alpha$-helices [22] (Figures 4 and S5-S7). Like other biosynthetic thiolases, $\operatorname{ReBktB}$ is a tetramer in which residues 120-143 from each monomer contribute to an eight-stranded, antiparallel $\beta$-barrel. Soaking of crystals with CoA and acetyl-CoA was attempted; however, samples rapidly deteriorated in their presence. Crystal structures were superposed using PyMol to compare thiolase active sites [23] (Figure 5).

\subsection{Rational engineering}

The M290A mutant catalyzed the retro-Claisen condensation of (2RS)-2-methyl-3-oxopentanoyl$S$-NAC (5), albeit complete conversion in $24 \mathrm{~h}$ required $25 \mu \mathrm{M} R e$ BktB (Figure 6). Only trace activity was noted for wild-type and the M290L mutants in the same conditions. The M290A and M290L mutants showed greatly decreased activity towards 3-oxopentanoyl-S-NAC (2) compared to wild-type ReBktB (Figure S8).

\section{Discussion}

ReBktB has shown biocatalytic potential in vivo, aiding in the production of valuable chiral building blocks [10]. In order to realize the biocatalytic potential of ReBktB in vitro, alter its substrate specificity, and enhance its catalytic properties, interactions between potential substrates and regions of ReBktB need to be more fully characterized - especially the CoA-binding region, the acyl group binding 
pocket for the priming acyl-CoA, and the acyl group binding pocket for the extending acyl-CoA (Figure $4 a)$.

That ReBktB can catalyze retro-Claisen reactions on NAC-linked substrates reveals that the residues contacting the adenosine portion of CoA are not essential for catalysis, even though they likely enhance catalytic efficiency (Figure 5b). By analogy with the CoA-bound structures of $Z r P h b A$ (PDBs 1DLV and 1QFL) [12,13], when an acyl-CoA is bound by ReBktB its adenine ring is sandwiched between R222 and L232 and its ribose 2'-OH forms a charged hydrogen bond with the R222 guanidinium. Since the CoA pantetheinyl arm of the priming and extending acyl-CoA substrates are differentially positioned during catalysis, the CoA tunnel accommodates at least two conformations of the pantetheinyl moiety. That the CoA binding tunnel does not tightly bind the pantetheinyl moiety is also indicated by crystal structures of CoA-bound thiolases (PDBs 1DLV, 1DM3, 1QFL, 1M10, and 4O9C) $[12,13,24,25]$. Thus, the catalytic efficiency of ReBktB towards NAC-linked substrates may suffer most in comparison with CoA-linked substrates due to the relative loss of interactions with the CoA adenosine moiety.

By analogy with $\mathrm{ZrPhbA}$, when the priming acyl-CoA is positioned for the transthioesterification reaction the thioester carbonyl forms hydrogen bonds with C90 NH and G382 NH (Figure 4b). If an acyl group bound to $R e \mathrm{BktB}$ points in the same direction as the acetyl group in the crystal structure of acetylated $\mathrm{ZrPhbA}$ (PDB 1DM3) [13], it would be located in a hydrophobic pocket created, in part, by Y66' (the prime indicates a residue from the adjacent monomer) and $\alpha 5$. The equivalent pocket in ZrPhbA, partially constructed by Q64' and $\alpha 5$, is smaller. The side chain of the conserved loop residue Q64' in $\mathrm{ZrPhbA}$ is closer to the catalytic residues compared to the side chain of helix-embedded Y66' of $\operatorname{ReBktB}$ (Figure 4c). Both residues form hydrogen bonds with the C-terminal end of $\alpha 5$; however, because $\alpha 5$ is narrower in $\operatorname{ReBktB}$ (being one residue shorter) it creates more space for the acyl group of the priming acyl-CoA. 
A recent crystal structure reveals the active site architecture of $\operatorname{RePhbA}$ (PDB 4O9C) [25] to be nearly identical to that of $\mathrm{ZrPhbA}$ (they share $63 \%$ sequence identity, while ReBktB and $\operatorname{RePhbA}$ only share 52\% sequence identity) [21], with the conserved glutamine and $\alpha 5$ in equivalent positions (Figure 5a). The small pocket they create may explain why both of these biosynthetic thiolases are specific for acetyl-CoA as a priming acyl-CoA. Degradative thiolases, which often perform thiolysis on long $\beta$ ketoacyl substrates, do not contain the glutamine or $\alpha 5$ (e.g., PDBs 1AFW, 2WU9, and 3GOA, from the Saccharomyces cerevisiae peroxisome, the Arabidopsis thaliana peroxisome, and Salmonella typhimurium) [26,27] (Figure 4c). The region where the glutamine and $\alpha 5$ would be located in degradative thiolases is hypothesized to bind fatty acyl chains. In crystal structures of degradative thiolases from S. cerevisiae and A. thaliana (PDBs 1AFW and 2WU9) [26,27], 2-methyl-2,4-pentanediol and ethylene glycol are observed in this region, and in the crystal structure of a Mycobacterium tuberculosis degradative thiolase a steroid substrate mimic can be observed (PDB 4UBT) [28].

We sought to better understand interactions between known ReBktB substrates and the ReBktB active site. The presented 2.01-Å resolution crystal structure provides a unique opportunity to visualize the conformations available to active site residues since the asymmetric unit possesses 16 crystallographically-distinct monomers. The side chains of L89 and M158 show more conformational freedom compared to the side chains of the catalytic residues C90, H350, and C380, which are observed in the same orientation within each monomer (Figure 5b). Since ReBktB can accept acetyl-, propionyl-, glycolyl-, butyryl-, isobutyrl-, and valeryl-CoA as priming substrates, the orientations that these acyl groups adopt within the active site were investigated. After placing the first two carbons of a valeryl group as observed for the acetyl group bound to $\mathrm{ZrPhbA}$ and rotating the $\mathrm{C}_{\alpha}-\mathrm{C}_{\beta}$ torsion angle $\sim 40^{\circ}$ as in several acyl-enzyme complexes of the thiolase superfamily (PDBs 1EK4, 1M4T, 1TQY, 4NA2, 4NA3) [23, 29-31], the next three carbons were modeled such that no clashes were made either with side chains or a bound acetyl-CoA (Figure 5c). As modeled, the valeryl group makes hydrophobic contact with the 
side chains of V57, L89, A148, M158, and I352. Shorter acyl chains may bind similarly; the glycolyl hydroxyl group would be in position to form a hydrogen bond with the A148 carbonyl.

The superposition of biosynthetic thiolases with $R e \mathrm{BktB}$ shows that the active site region where the extending acetyl-CoA is bound during carbon-carbon bond-formation is equivalent in all three enzymes (Figure 5a). A methionine (M290 in $R e \mathrm{BktB})$ is positioned to make hydrophobic contact with the acetyl methyl group, as observed in the crystal structure of the acetylated $\mathrm{ZrPhbA}$ in complex with acetyl-CoA (PDB 1DM3) [13]. That M290 was observed in the same conformation in each of the 16 crystallographically-distinct $R e \mathrm{BktB}$ monomers may indicate the importance of this residue in naturally selecting against extending acyl-CoA's larger than acetyl-CoA (Figure 4b). Interestingly, ReBktB does not exclude glycolyl-CoA as an extending acyl-CoA but does exclude propionyl-CoA [10].

In an attempt to engineer ReBktB to generate $\alpha$-methyl substituted products, M290A and M290L mutants were expressed, purified, and assayed (Figure 6). At a concentration of $25 \mu \mathrm{M}, \mathrm{M} 290 \mathrm{~A}$ catalyzes the complete thiolysis of (2RS)-2-methyl-3-oxopentanoyl-S-NAC (5) in $24 \mathrm{~h}$. At the same concentration, wild-type $R e B k t B$ and the M290L mutant only show trace activity. Both the M290A and M290L mutants show greatly diminished activity toward 3-oxopentanoyl-S-NAC (2) compared to the wild-type enzyme (Figure S8). These results indicate that further rational engineering to tune the substrate specificity of $\operatorname{ReBktB}$ is possible.

\section{Conclusion}

The presented structural and functional analysis will be useful in engineering desired biocatalytic properties into ReBktB through rational or directed evolution approaches. Mutating V57, L89, A148, M158, and I352 to smaller residues may increase the range of priming acyl thioesters accepted by ReBktB and allow the incorporation of synthetically-useful functional groups. As suggested by the M290A mutant reported here, substitution of M290 by smaller residues may enable the condensation of extending acyl 
thioester substrates other than acetyl and glycolyl thioesters to generate $\alpha$-substituted, $\beta$-ketoacyl thioesters. Such products could be reduced in a stereocontrolled manner (e.g., by modular polyketide synthase ketoreductases) to yield libraries of two-stereocenter chiral building blocks (Figure 1c) [32]. That $R e \mathrm{BktB}$ is active towards NAC-linked substrates enables its usage in practical in vitro biocatalytic schemes, which would be attractive since such reactions contain fewer components and are more controllable than those in vivo. Engineering greater extending acyl thioester promiscuity within $R e \mathrm{BktB}$ could result in mixtures of reaction products; however, valuable small molecule products generated from such in vitro biocatalytic schemes could be chromatographically resolved or selectively utilized by downstream enzymes. In the current advent of biosynthetic biocatalysis, the studies reported here will aid in engineering and utilizing $R e \mathrm{BktB}$ to generate valuable molecules from abundant precursors.

\section{Materials and methods}

\subsection{Cloning, expression, and purification of ReBktB}

The $R e B k t B$ gene was amplified from the $\mathrm{pCDF} / \mathrm{pct} / \mathrm{tes} \mathrm{B}$ plasmid [10] with primers $5 \square$ ATGCTTGCAcatatgACGCGTGAAGTGGTAGTGGTA-3 $\square \quad 5 \square-$ GTACGAACGgaattcTCAGATACGCTCGAAGATGGCGGC-3 $\square$ (restriction sites in lower case; stop codon underlined), digested with $N d e \mathrm{I}$ and EcoRI, and ligated into pET28b (Novagen). E. coli BL21(DE3) cells carrying this plasmid were grown in LB media with $50 \mathrm{mg} / \mathrm{L}$ kanamycin at $37^{\circ} \mathrm{C}$. Upon reaching $\mathrm{OD}_{600}=0.4$, cultures were cooled to $15^{\circ} \mathrm{C}$ and induced with $0.5 \mathrm{mM}$ IPTG. After $17 \mathrm{~h}$, cells were pelleted, resuspended in lysis buffer (500 mM NaCl, $30 \mathrm{mM}$ HEPES pH 7.5, 10\% (v/v) glycerol) and sonicated. Cell lysate was centrifuged at $30,000 \times g$ for $1 \mathrm{~h}$, and the supernatant was poured over a column of Ni-NTA beads (Expedeon) equilibrated with lysis buffer. Beads were washed with 10 column volumes of $15 \mathrm{mM}$ imidazole in lysis buffer and protein was eluted with 1.5 column volumes of $150 \mathrm{mM}$ 
imidazole in lysis buffer. Eluate was further purified on a Superdex 200 gel filtration column (GE Healthcare Life Sciences) equilibrated with $150 \mathrm{mM} \mathrm{NaCl}, 10 \mathrm{mM}$ HEPES pH 7.5, 10\% (v/v) glycerol. Column fractions were exchanged in $25 \mathrm{mM} \mathrm{NaCl}, 10 \mathrm{mM}$ HEPES pH 7.5, $10 \%$ (v/v) glycerol and brought to $37 \mathrm{mg} / \mathrm{mL}$ in a centrifugal concentrator. Aliquots were flash frozen in liquid nitrogen and stored at $-80{ }^{\circ} \mathrm{C}$ until further use. The isolated yield of $R e B k t B$ was $\sim 10 \mathrm{mg} / \mathrm{L}$ culture.

Mutations of $R e \mathrm{BktB}$ were generated by PCR amplification of the construct described above using primers 5'-GTGGACCCGAAGGCCGCCGGCATCGGCCCGGTG-3' and 5'CACCGGGCCGATGCCCGGGGCCTTCGGGTCCAC-3' for M290A, and primers 5'CGTGGACCCGAAGGCCCTGGGCATCGGCC-3' and 5'-GGCCTTCGGGTCCACGCCGGCAT-3' for M290L. The resulting products were digested with DpnI to remove the template plasmid and subsequently transformed into E. coli BL21(DE3) cells. Expression and purification of the ReBktB mutants followed that of the wild-type enzyme.

\subsection{Syntheses of NAC and $\beta$-ketoacyl-S-NAC substrates}

Syntheses of NAC, 1, 2, 3, and $\mathbf{5}$ were carried out as described [32]. Syntheses of $\mathbf{4}$ and $\mathbf{6}$ were carried out as described [33].

\subsection{In vitro thiolytic cleavage of $\beta$-ketoacyl-S-NAC substrates}

Enzyme aliquots were rapidly thawed and placed on ice. $\operatorname{ReBktB}(1 \mu \mathrm{M}$ final) was added to 100 $\mathrm{mM} \mathrm{NaCl}, 100 \mathrm{mM}$ HEPES pH 7.5, 10\% (v/v) glycerol, $5 \mathrm{mM} \mathrm{NAC}$, and $1 \mathrm{mM} \mathrm{1,} \mathrm{2,} \mathrm{3,} \mathrm{4,} \mathrm{or} 5$ in a total volume of $1 \mathrm{~mL}$ in triplicate. Negative controls lacking either ReBktB or NAC were also prepared. After incubating for 1 or $24 \mathrm{~h}$ at $22{ }^{\circ} \mathrm{C}$, quenching was effected by mixing $190 \mu \mathrm{L}$ reaction solution with $10 \mu \mathrm{L}$ $7 \mathrm{M} \mathrm{HCl}$. Negative controls lacking both $\mathrm{ReBktB}$ and $\mathrm{HCl}$ were also prepared to ensure that quenching did not promote acid-catalyzed hydrolysis of thioester substrates. Samples were centrifuged and their 
supernatants were analyzed at $235 \mathrm{~nm}$ by reverse-phase HPLC on a Varian Microsorb-MV 300-5 C18 $250 \times 4.6 \mathrm{~mm}$ column (Agilent Technologies; 50- $\mu \mathrm{L}$ injection loop) and Waters 2998 PDA detector. Mobile phases consisted of $0.1 \%$ TFA in water (solution A) and $0.1 \%$ TFA in methanol (solution B). Reactions containing 1 were injected $(20 \mu \mathrm{L})$ on a 30-min linear gradient of $0-20 \%$ solution B with a flow rate of $1 \mathrm{~mL} / \mathrm{min}$. Reactions containing $\mathbf{2 , 3 , 4}$, or $\mathbf{5}$ were injected $(20 \mu \mathrm{L})$ on a 30 -min linear gradient of $5-50 \%$ solution B with a flow rate of $1 \mathrm{~mL} / \mathrm{min}$. Peaks for product $\mathbf{6}$, monitored at $235 \mathrm{~nm}$, eluted at 17.3 min for reactions containing $\mathbf{1}$ or 10.2 min for reactions containing $\mathbf{2}, \mathbf{3}$, or $\mathbf{4}$ (Figures 2 and S1). Peaks for product 6 were integrated in Breeze 2 software (Waters) and their areas were compared to those of a standard curve of 0.1-1 mM 6 to determine concentrations (Figure S2). For reactions containing 5, peaks for product 7, which eluted at $15.3 \mathrm{~min}$, were monitored at $235 \mathrm{~nm}$ and integrated. Background from reaction solutions lacking enzyme was subtracted from peak areas, and calculated concentrations were adjusted for dilution factor. For reactions containing 1, peak areas for product $\mathbf{6}$ were divided by 2 for direct comparison with other reactions. Product fractions were collected and further analyzed by positiveESI LC/MS (Agilent Technologies 1200 Series HPLC with a Gemini C18 5 micron $2.1 \times 50$ mm column coupled to an Agilent Technologies 6130 Series Quadrupole MS). Mobile phases consisted of 0.1\% formic acid in water (solution C) and $0.1 \%$ formic acid in acetonitrile (solution D). Samples were run on a 12-min linear gradient of 5-95\% solution $\mathrm{D}$ with a flow rate of $1 \mathrm{~mL} / \mathrm{min}$.

To compare the activities of ReBktB, the M290A mutant, and the M290L mutant toward 5, each enzyme was added at a concentration of $25 \mu \mathrm{M}$ to $100 \mathrm{mM} \mathrm{NaCl}, 100 \mathrm{mM}$ HEPES pH 7.5, 10\% (v/v) glycerol, $5 \mathrm{mM} \mathrm{NAC}$, and $1 \mathrm{mM} 5$ in a total volume of $0.5 \mathrm{~mL}$. To compare the activities of $R e \mathrm{BktB}$, the M290A mutant, and the M290L mutant toward 2, each enzyme was added at a concentration of $1 \mu \mathrm{M}$ to $100 \mathrm{mM} \mathrm{NaCl}, 100 \mathrm{mM}$ HEPES pH 7.5, 10\% (v/v) glycerol, $5 \mathrm{mM} \mathrm{NAC,} \mathrm{and} 1 \mathrm{mM} 2$ in a total volume of $1 \mathrm{~mL}$. In both assays samples were analyzed as above at 0,1 , and $24 \mathrm{~h}$. 


\subsection{Crystallization, data collection, processing, phasing, and refinement}

Enzyme aliquots were rapidly thawed and placed on ice. Crystals were grown by sitting-drop vapor diffusion by adding $1 \mu \mathrm{L}$ protein solution to $0.5 \mu \mathrm{L} 22 \%$ (w/v) PEG 4000, $100 \mathrm{mM}$ HEPES pH 7.0, $12 \%(\mathrm{v} / \mathrm{v})$ glycerol at $22{ }^{\circ} \mathrm{C}$. Crystals were harvested from the mother liquor and immediately flash frozen in liquid nitrogen without addition of cryoprotectants; they dissolved unless removed from the mother liquor within $48 \mathrm{~h}$ of drop preparation.

A 2.0- $\AA$ dataset was collected from a single crystal at $100 \mathrm{~K}$ at Advanced Light Source beamline 5.0.3 $(\lambda=0.9765 \AA)$ and indexed, integrated, and scaled in HKL2000 [34] (Table 1). Intensities were converted to amplitudes, and 5\% of scaled reflections were reserved for calculating $R_{\text {free }}$ in TRUNCATE [35] of the CCP4 software suite [19]. Phases were solved via molecular replacement by searching for eight copies of a Clostridium difficile thiolase (PDB 4DD5) dimer in space group P1 in Phaser [14] of CCP4. To convert side chains to those of the $R$. eutropha enzyme, a SCWRL model [36] obtained from the Fold \& Function Assignment System (FFAS) server [37] was superposed onto each monomer of the Phaser solution. Pseudo-merohedral twinning and associated operators were identified (Table S2) in phenix.xtriage of the PHENIX software bundle [16]. Iterative refinement was performed in Coot [38] and REFMAC5 [18] of CCP4 (with intensity-based twin refinement, distance and angle restraint weights of 4.0, and automatically generated local NCS restraints). Throughout refinement, the MolProbity server was consulted for structure validation [39]. Structure factor amplitudes and atomic coordinates for $R e B k t B$ were deposited in the Protein Data Bank with PDB ID 4W61.

\section{Acknowledgments}

We gratefully acknowledge Kristala L. J. Prather for the pCDF/pct/tesB plasmid, helpful discussions, and reviewing the manuscript. This project was supported by the Welch Foundation (F-1712) and the National Institutes of Health (GM106112). Instrumentation and technical assistance for this work 
were provided by the Macromolecular Crystallography Facility, with financial support from the College of Natural Sciences, the Office of the Executive Vice President and Provost, and the Institute for Cellular and Molecular Biology at the University of Texas at Austin. The Berkeley Center for Structural Biology is supported in part by the National Institutes of Health, National Institute of General Medical Sciences, and the Howard Hughes Medical Institute. The Advanced Light Source is supported by the Director, Office of Science, Office of Basic Energy Sciences, of the U.S. Department of Energy under Contract No. DE-AC02-05CH11231. 


\section{References}

[1] S. Masamune, C.T. Walsh, A.T. Sinskey, O.P. Peoples, Pure Appl. Chem. 16 (1989) 303-312.

[2] S. Thompson, F. Mayerl, O.P. Peoples, S. Masamune, A.J. Sinskey, C.T. Walsh. Biochemistry 28 (1989) 5735-5742.

[3] P.A. Holmes. Phys. Technol. 16 (1985) 32-36.

[4] A.J. Anderson, E.A. Dawes, Microbiol. Rev. 54 (1990) 450-472.

[5] S. Slater, K.L. Houmiel, M. Tran, T.A. Mitsky, N.B. Taylor, S.R. Padgette, K.J. Gruys, J. Bacteriol. 180 (1998) 1979-1987.

[6] H. Salehizadeh, M.C. van Loosdrecht, Biotechnol. Adv. 22 (2004) 261-279.

[7] R.A. Verlinden, D.J. Hill, M.A. Kenward, C.D. Williams, I. Radecka, J. Appl. Microbiol. 102 (2007) 1437-1449.

[8] S.P. Valappil, A.R. Boccaccini, C. Bucke, I. Roy, Antonie van Leeuwenhoek 91 (2007) 1-17.

[9] S.H. Lee, O.J. Park, Appl. Microbiol. Biotechnol. 84 (2009) 817-828.

[10] C.H. Martin, H. Dhamankar, H.C. Tseng, M.J. Sheppard, C.R. Reisch, K.L. Prather, Nat. Commun. $4(2013) 1-9$

[11] J.T. Davis, R.N. Moore, B. Imperiali, A.J. Pratt, K. Kobayashi, S. Masamune, A.J. Sinskey, C.T. Walsh, T. Fukui, K. Tomita, J. Biol. Chem. 262 (1987) 82-89. 
[12] Y. Modis, R.K. Wierenga, Structure 7 (1999) 1279-1290.

[13] Y. Modis, R.K. Wierenga, J. Mol. Biol. 297 (2000) 1171-1182.

[14] A.J. McCoy, R.W. Grosse-Kunstleve, P.D. Adams, M.D. Winn, L.C. Storoni, R.J. Read, J. Appl. Cryst. 40 (2007) 658-674.

[15] T.O. Yeates, B.C. Fam, Structure 7 (1999) R25-R29.

[16] P.D. Adams, P.V. Afonine, G. Bunkóczi, V.B. Chen, I.W. Davis, N. Echols, J.J. Headd, L.W. Hung, G.J. Kapral, R.W. Grosse-Kunstleve, A.J. McCoy, N.W. Moriarty, R. Oeffner, R.J. Read, D.C. Richardson, J.S. Richardson, T.C. Terwilliger, P.H. Zwart, Acta Crystallogr. Sect. D 66 (2010) 213221.

[17] J.E. Padilla, T.O. Yeates, Acta Crystallogr. Sect. D 59 (2003) 1124-1130.

[18] G.N. Murshudov, A.A. Vagin, E.J. Dodson, Acta Crystallogr. Sect. D 53 (19997) 240-255.

[19] M.D. Winn, C.C. Ballard, K.D. Cowtan, E.J. Dodson, P. Emsley, P.R. Evans, R.M. Keegan, E.B. Krissinel, A.G. Leslie, A. McCoy, S.J. McNicholas, G.N. Murshudov, N.S. Pannu, E.A. Potterton, H.R. Powell, R.J. Read, A. Vagin, K.S. Wilson, Acta Crystallogr. Sect. D 67 (2011) 235-242.

[20] E.J. Kim, H.F. Son, S. Kim, J.W. Ahn, K.J. Kim. Biochem. Biophys. Res. Commun. 444 (2014) 365-369. 
[21] F. Sievers, A. Wilm, D. Dineen, T.J. Gibson, K. Karplus, W. Li, R. Lopez, H. McWilliam, M. Remmert, J. Söding, J.D. Thompson, D.G. Higgins, Mol. Syst. Biol. 7 (2011) 539-544.

[22] A.M. Haapalainen, G. Meriläinen, R.K. Wierenga, Trends Biochem. Sci. 31 (2006) 64-71.

[23] The PyMOL Molecular Graphics System, Version 1.7.4 Schrödinger, LLC.

[24] P. Kursula, J. Ojala, A.M. Lambeir, R.K. Wierenga. Biochemistry 41 (2002)15543-15556.

[25] E.J. Kim, K.J. Kim. Biochem. Biophys. Res. Commun. 452 (2014) 124-129.

[26] M. Mathieu, Y. Modis, J.P. Zeelen, C.K. Engel, R.A. Abagyan, A. Ahlberg, B. Rasmussen, V.S. Lamzin, W.H. Kunau, R.K. Wierenga. J. Mol. Biol 273 (1997) 714-728.

[27] V.E. Pye, C.E. Christensen, J.H. Dyer, S. Arent, A. Henriksen, J. Biol. Chem. 285 (2010) 2407824088.

[28] C.M. Schaefer, R. Lu, N.M. Nesbitt, J. Schiebel, N.S. Sampson, C. Kisker, Structure 23 (2015) 21 33.

[29] J.G. Olsen, A. Kadziola, P. von Wettstein-Knowles, M. Siggaard-Andersen, S. Larsen, Structure 9 (2001) 233-43.

[30] A.T. Keatinge-Clay, D.A. Maltby, K.F. Medzihradsky, C. Khosla, R.M. Stroud, Nat. Struct. Mol. Biol. 11 (2004) 888-93. 
[31] D.C. Gay, G. Gay, A.J. Axelrod, M. Jenner, C. Kohlhaas, A. Kampa, N.J. Oldham, J. Piel, A.T. Keatinge-Clay, Structure 22 (2014) 444-51.

[32] S.K. Piasecki, C.A. Taylor, J.F. Detelich, J. Liu, J. Zheng, A. Komsoukaniants, D.R. Siegel, A.T. Keatinge-Clay, Chem. Biol. 18 (2011) 1331-1340.

[33] A.J. Hughes, J.F. Detelich, A.T. Keatinge-Clay, Med. Chem. Comm. 3 (2011) 956-959.

[34] Z. Otwinowski, W. Minor, Methods Enzymol. 276 (1997) 307-326.

[35] S. French, K. Wilson, Acta Crystallogr Sect A 34 (1978) 517-525.

[36] G.G. Krivov, M.V. Shapovalov, R.L. Dunbrack Jr., Proteins 77 (2009) 778-795.

[37] L. Jaroszewski, Z. Li, X.H. Cai, C. Weber, A. Godzik, Nucleic Acids Res. 33 (2005) W284-W288.

[38] P. Emsley, K. Cowtan, Acta Crystallogr. Sect. D 60 (2004) 2126-2132.

[39] V.B. Chen, W.B. Arendall 3rd, J.J. Headd, D.A. Keedy, R.M. Immormino, G.J. Kapral, L.W. Murray, J.S. Richardson, D.C. Richardson, Acta Crystallogr. Sect. D 66 (2010) 12-21. 


\section{FIGURE LEGENDS}

Figure 1. Natural and engineered pathways utilizing biosynthetic thiolases. (a) Polyhydroxyalkanoate (PHA) synthesis. After the biosynthetic thiolase PhbA or BktB condenses two acyl-CoA's into a $\beta$ ketoacyl-CoA, PhbB can generate a $R$ - $\beta$-hydroxy butyryl monomer for PhbC-mediated addition to a growing PHA polymer. (b) In vivo pathway engineered to yield $\beta$-hydroxy acids. After condensation and reduction by BktB and $\mathrm{PhbB}$, respectively, the thioesterase TesB releases $\beta$-hydroxy acid chiral building blocks. (c) Proposed in vitro pathway. Modular polyketide synthase ketoreductases (PKS KRs) coupled with an NADPH-regeneration system could both drive the production of $\alpha$-substituted, $\beta$-ketoacyl-SNAC's by BktB as well as set the stereochemistry of $\alpha$ - and $\beta$-substituents of generated $\alpha$-substituted, $\beta$ hydroxyacyl chiral building blocks [31].

Figure 2. In vitro thiolytic cleavage of $\beta$-ketoacyl-S-NAC thioesters by ReBktB. (a) Reactions assayed with $R e \mathrm{BktB}$. R-groups of $\beta$-ketoacyl-S-NAC substrates and acyl-S-NAC products are indicated. ReBktB did not catalyze the thiolysis of compound 5. (b) Chromatograms $(235 \mathrm{~nm}$ ) of reactions containing 1 (left panel) and 2, 3, or 4 (right panel; overlay) $(1 \mu \mathrm{M} R e$ BktB was incubated with $1 \mathrm{mM} \beta$-ketoacyl-S-NAC and $5 \mathrm{mM}$ NAC for $1 \mathrm{~h}$ at $22{ }^{\circ} \mathrm{C}$, then acid-quenched and analyzed by $\mathrm{C}_{18}$ reverse-phase HPLC). A shallower gradient was employed for reactions containing 1 to obtain better resolution. (c) $\operatorname{ReBktB}$ preference: $\mathbf{2}>\mathbf{1}>\mathbf{3} \sim \mathbf{4}$. The concentration of product 6 was determined by integrating the area of peak 6, subtracting that of corresponding $-R e \mathrm{BktB}$ controls, and accounting for dilution factor $(0.873 \pm 0.031$ $\mathrm{mM}$ for $\mathbf{2}, 0.238 \pm 0.007 \mathrm{mM}$ for $\mathbf{1}, 0.089 \pm 0.006 \mathrm{mM}$ for $\mathbf{3}$, and $0.082 \pm 0.005 \mathrm{mM}$ for 4 ). For reactions containing 1, peak areas for product $\mathbf{6}$ were divided in half. Error bars indicate standard deviation of triplicate measurements. 
Figure 3. Crystal structure of $R e \mathrm{BktB}$. (a) The asymmetric unit of the ReBktB crystal contains four tetramers (distinguished by color). The $P 1$ unit cell is displayed as a wire box. (b) Stereoview of superposed ReBktB (green) and CoA-bound ZrPhbA (cyan, PDB 1DLV). C $\alpha$ r.m.s.d. = $0.64 \AA$ over 339 residues.

Figure 4. Active site elements of ReBktB. (a) Condensation and thiolysis reactions (clockwise and counterclockwise, respectively). Select atoms are shown from substrates, products, C90, H350, C380, and G382. Binding pockets for CoA, $R_{1}$, and $R_{2}$ are drawn as red, green, and blue semicircles, respectively. (b) Stereoview of superposed active sites of ReBktB (light and dark green chains) and acetyl-CoA-bound acetyl-ZrPhbA (cyan and blue chains; PDB 1DM3). ReBktB possesses unique features in helices $\alpha 3$ (kinked at $\mathrm{Y} 66$ ) and $\alpha 5$ (narrower) that expand the $\mathrm{R}_{1}$ binding pocket relative to $\mathrm{ZrPhbA}$. The loop following $\alpha 5$ has been hidden for clarity (indicated with black circles). (c) Alignment of thiolase sequences corresponding to ReBktB helices $\alpha 3$ and $\alpha 5$ (residues 62-75 and 144-149, ReBktB numbering). PhbA-type biosynthetic thiolases, but not $R e B k t B$, share strong conservation in these regions, while degradative thiolases possess different architectures in these regions. Biosynthetic thiolases: R. eutropha BktB (NCBI YP_725948); R. eutropha PhbA (P14611); Z. ramigera PhbA (P07097); Pseudomonas nitroreducens (WP_017518929); Lamprocystis purpurea (WP_020506577); Thiothrix nivea (WP_002708073); C. difficile (Q18AR0); Mus musculus cytosolic (NP_033364); H. sapiens mitochondrial (P24752); and S. cerevisiae cytosolic (XXBYAC). Degradative thiolases: S. typhimurium (P0A2H7); Yersinia pestis (A4TR28); Achromobacter xylosoxidans (WP_020928315); Leptonema illini (WP_002774022); A. thaliana peroxisomal (Q56WD9); Glycine max (NP_001237076); Macaca mulatta peroxisomal (AFH32148); S. cerevisiae peroxisomal (P27796); Trypanosoma brucei (Q57XD5); and Aspergillus niger (EHA20372). Sequences were aligned with Clustal Omega [21].

Figure 5. Superposition of thiolase active sites validates differing substrate specificities. (a) Superposed stereoview of $\mathrm{ZrPhbA}$ (cyan carbons, with acetyl-CoA and enzyme-bound acetyl carbons in white; PDB 
1DM3), RePhbA (orange carbons; PDB 4O9A), and ReBktB (green carbons). (b) Superposed stereoview of $\mathrm{ZrPhbA}$ and the sixteen $\operatorname{ReBktB}$ monomers from the unit cell. L89 and M158 adopt more conformations than neighboring residues. (c) Model of acetyl-CoA-bound, valeryl-ReBktB intermediate. The valeryl group appears to fit within a hydrophobic pocket with proper geometry and no serious steric clashes.

Figure 6. The rationally-engineered M290A mutant is active toward an $\alpha$-substituted substrate. (a) Wildtype ReBktB, (b) the M190A mutant, and (c) the M190L mutant incubated with (2RS)-2-methyl-3oxopentanoyl-S-NAC (5) (25 $\mu \mathrm{M}$ enzyme; 0, 1, and $24 \mathrm{~h}$ timepoints). Significant thiolysis to propionyl-SNAC (7) is observed for the M190A mutant. 
a Biosynthesis of PHAs

b Engineered in vivo pathway

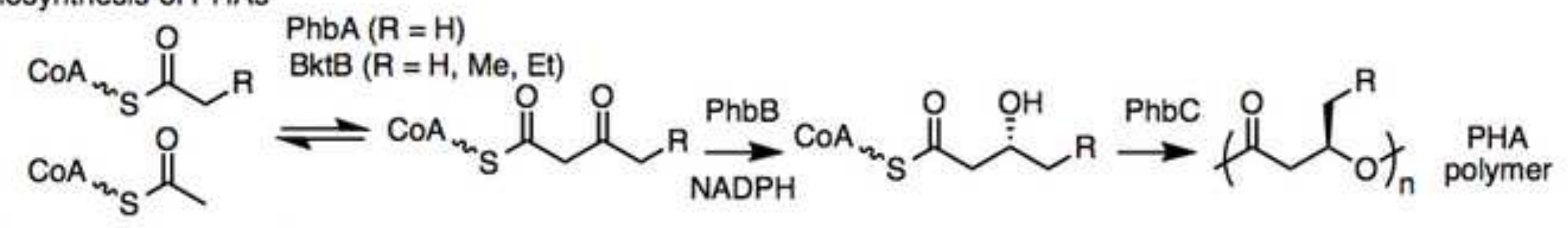

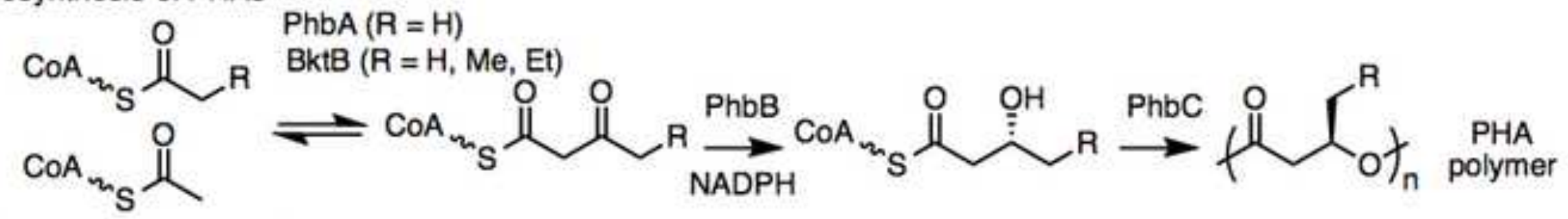

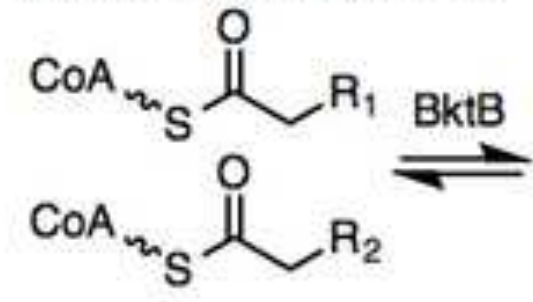

C Potential in vitro pathway

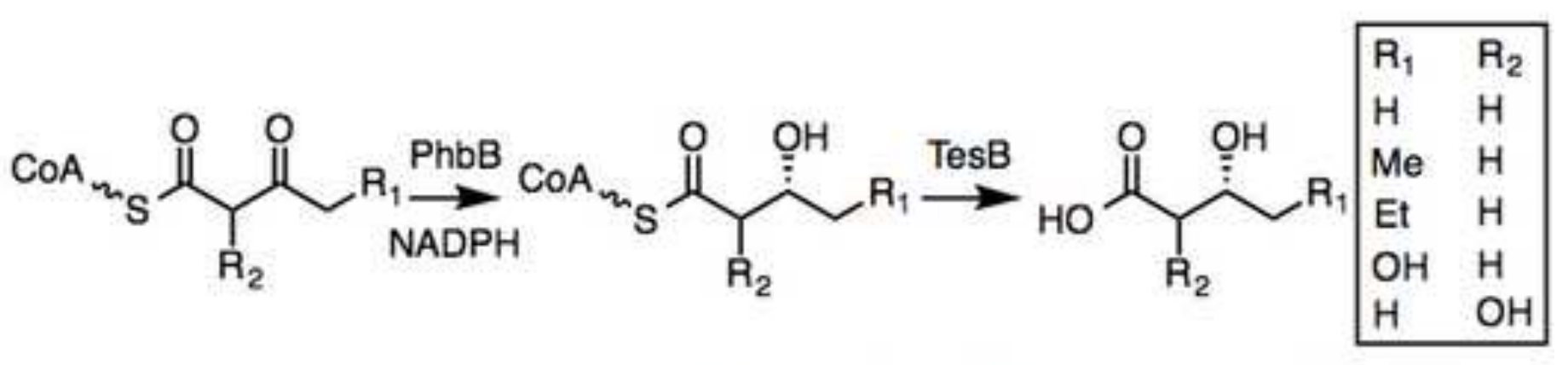<smiles></smiles> 
a<smiles>[R]C(=O)CC(=O)SCCNC(C)=O</smiles><smiles>[R]C(=O)SCCNC(C)=O</smiles>

substrate

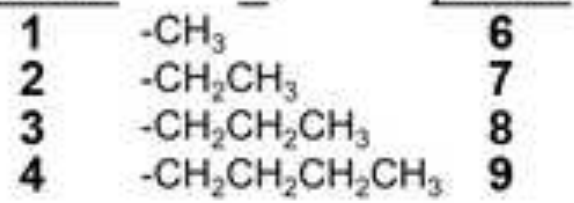

b

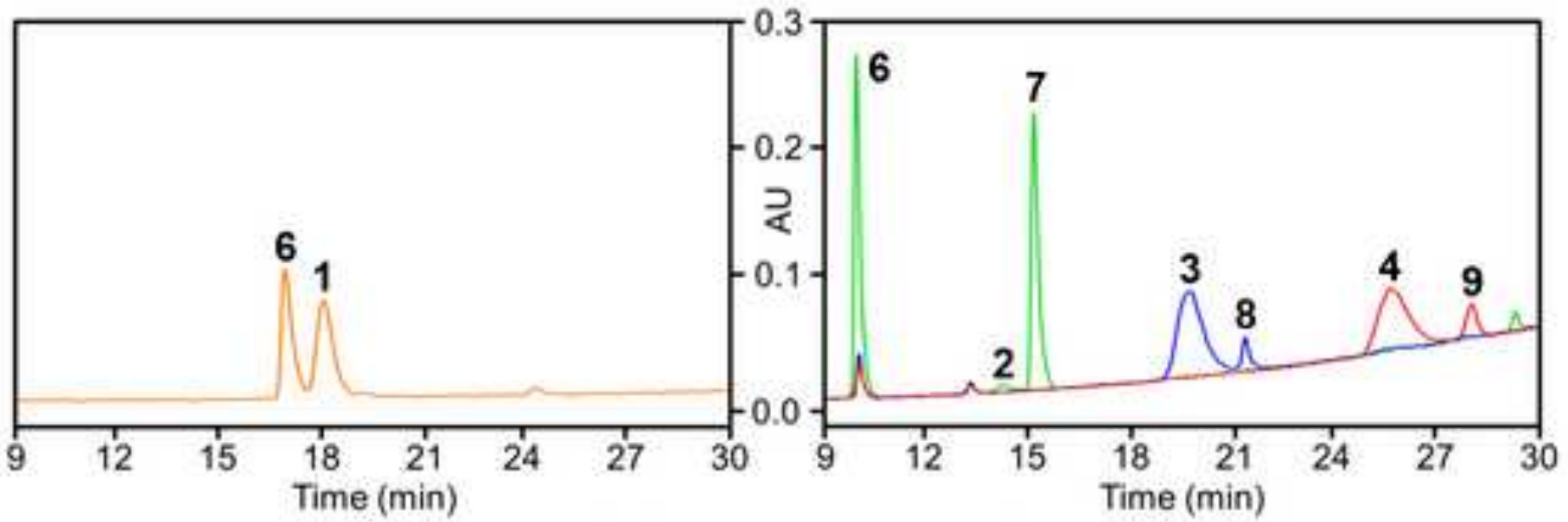

C

ReBktB-catalyzed thiolysis of
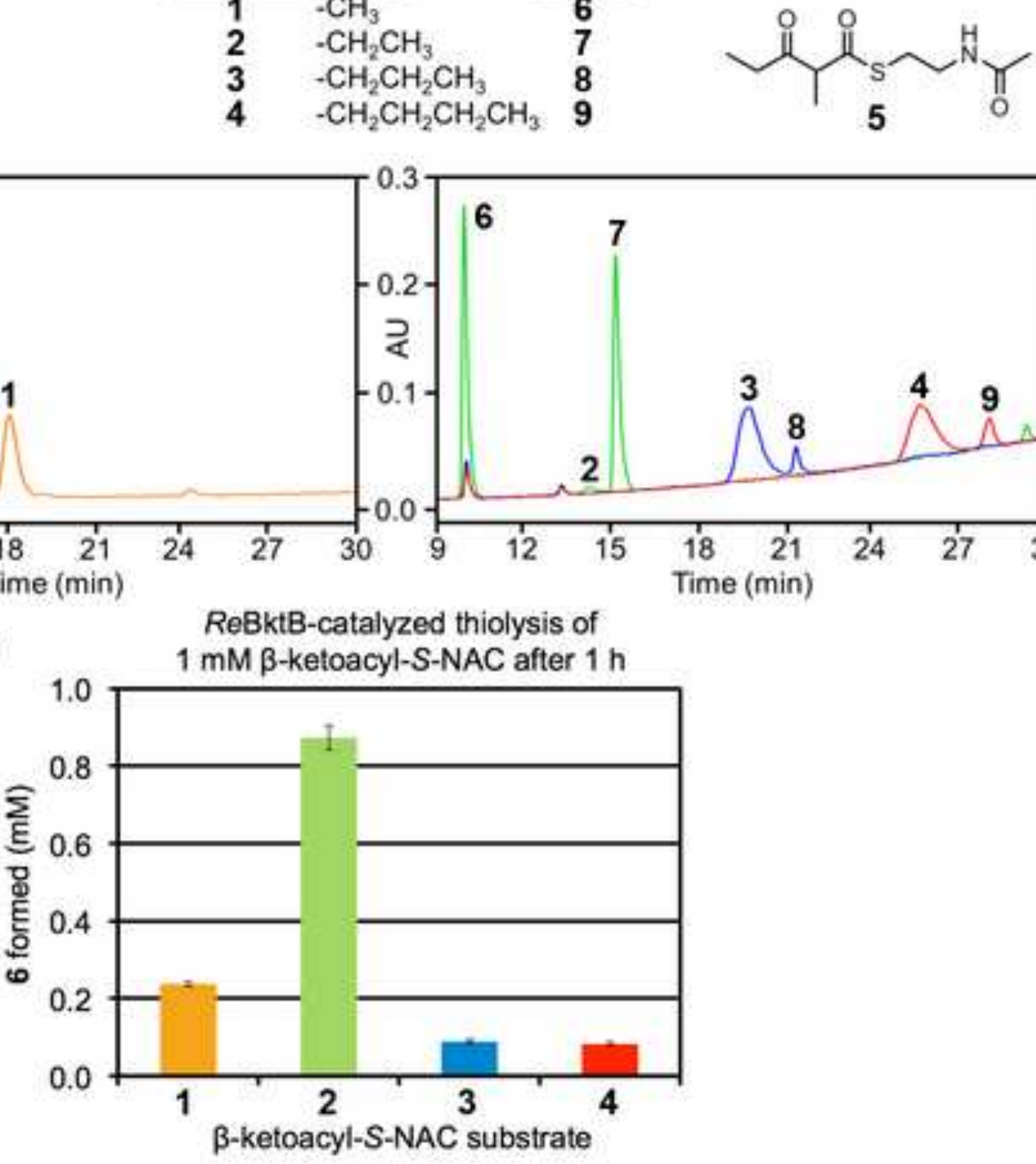

ime (min) 
a

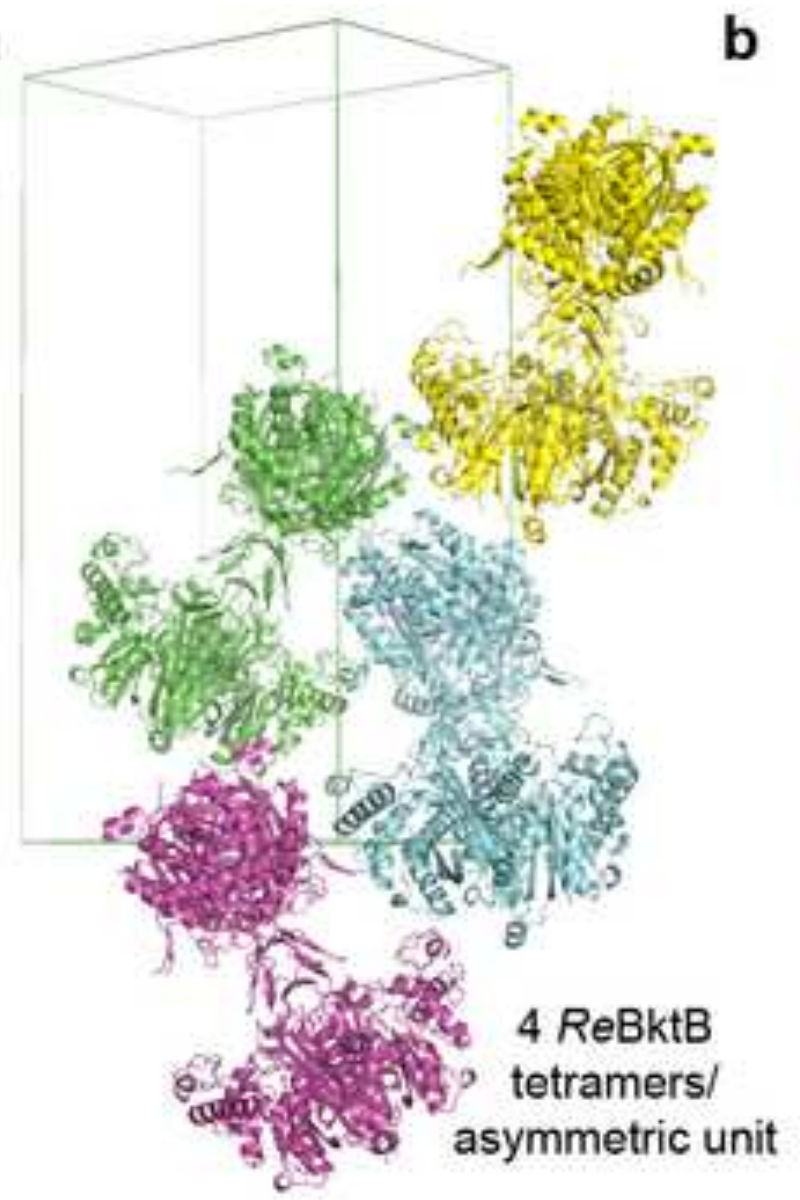

b

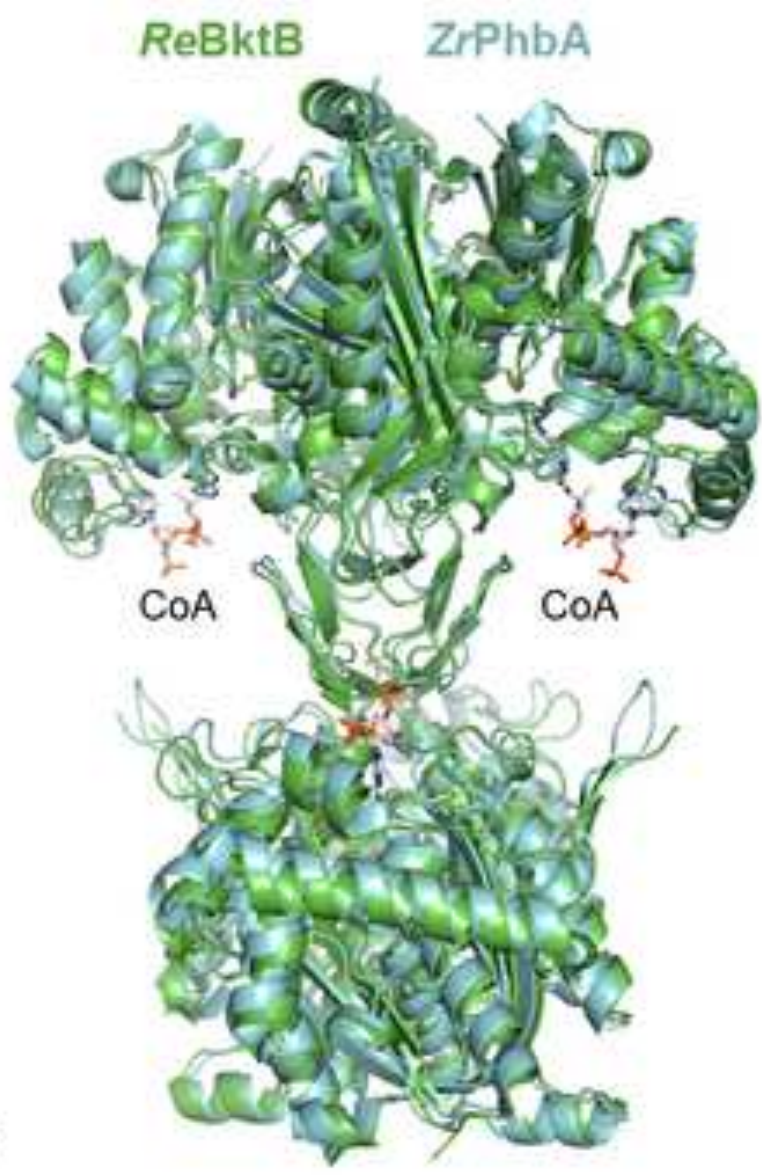

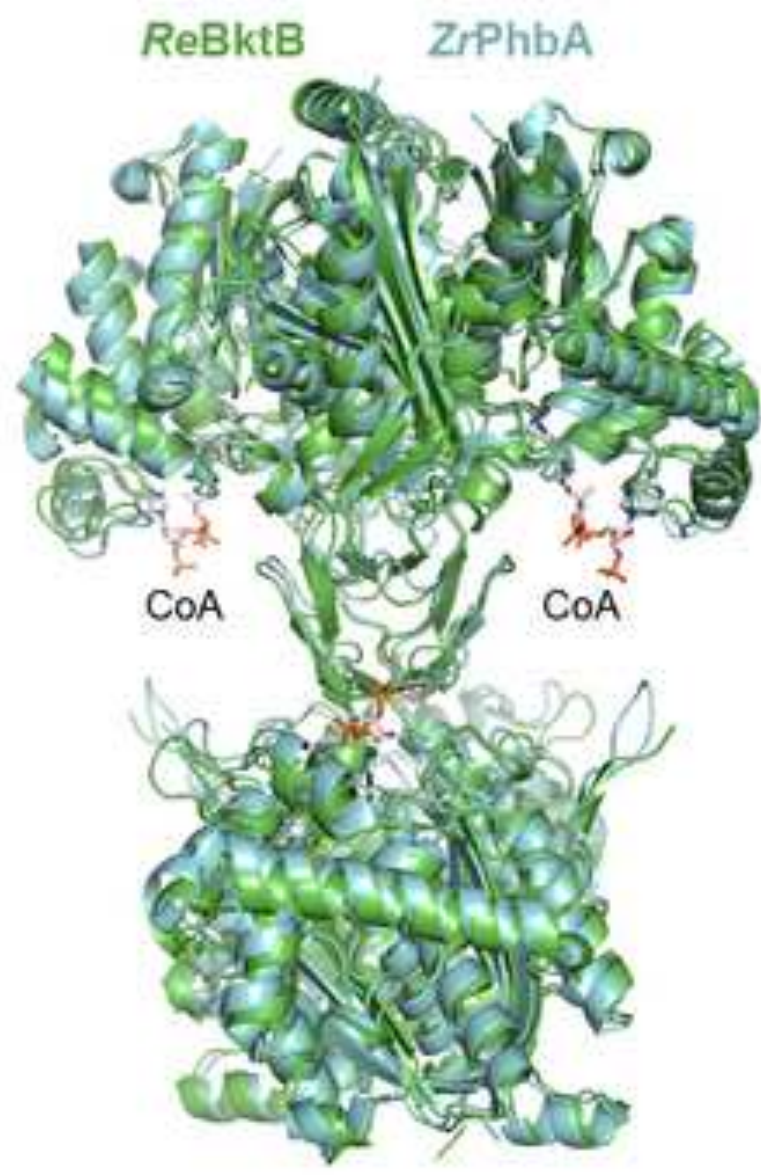


a

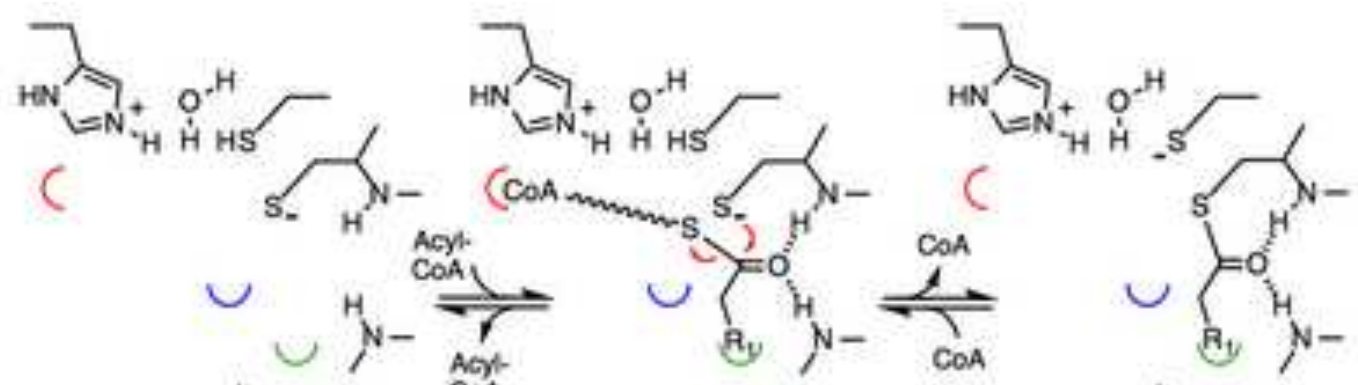

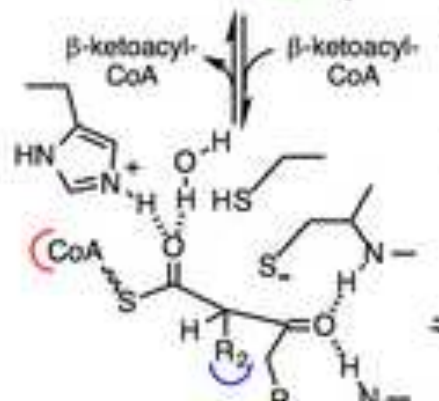
$\operatorname{CoA}$

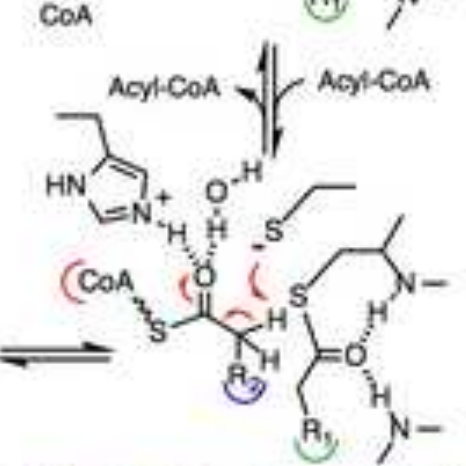

b<smiles>CCC1=C[NH+]=C[PH-]=[NH+]1</smiles>

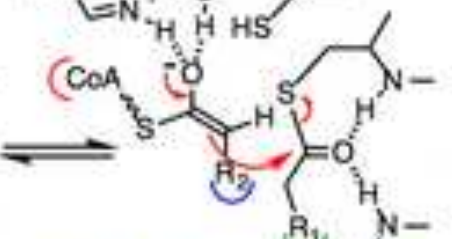

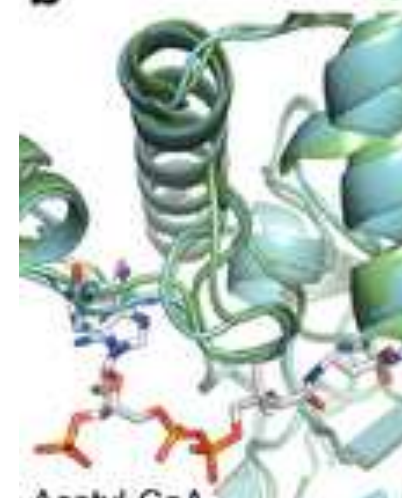

Acetyl-COA Bi $N-$
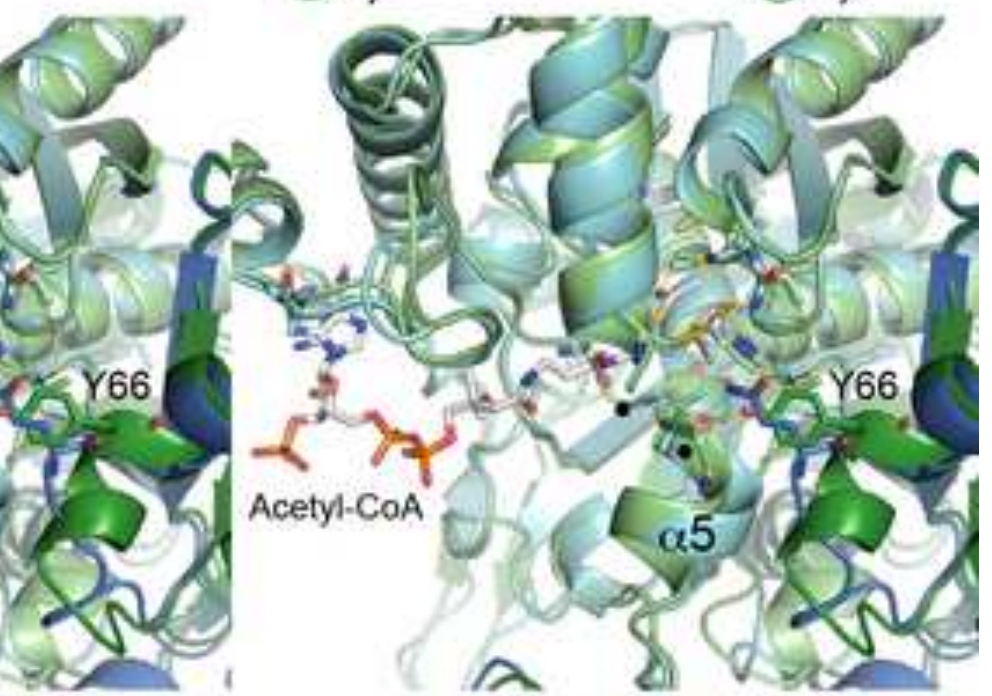

Acetyl-CoA

a5

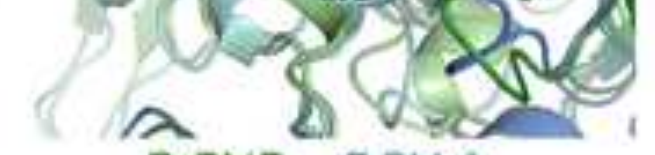

ReBktB

ZrPhbA

C
R. eutropha BktB VIQTEPRDMYLGRVAAVNGG

R. eutropha PhDA VLTAG-SGQNPARQAAIKAG Z. ramigera VLPAG - BGQNPARQAAMKAG P. nutroroducons TLTAG-AGQNPARQASIAAG L. pupuma VLTAG-VGQNPARQTAIKAG T. nivea VLTAG-VGQNPARQAVLSAG

c. dificile VLTAG-LGQNIARQIALGAG $M$. musculus cyto VLTAG-CGQNPTRQASVGAG $H$. sapvens mito VLQGG-BGQAPTRQAVLGAG S. cerevisiae cyto VLSAN - LGQAPARQVALTAG

S. Dyphomunium VQQTLEQGFNIARNAALLAE $Y$ pestis VQQTLEQGFNIARNASLLAE A. xylosoxidans VSQAGEQGANI GRLSALLAG $L$ iini VSQVGEQGACIARNAVLAAK A. thaliana perox VLAPGSQRASECRMAAFYAG G. max VLAPGSDRGIECRMAAFYAG M. mulatta VLQPGAG - A IMARIAQFLSD S. cerevisiae perox VLNVGAG - ATEHRAACLASG T. Druce FLGBLFSSQGHLGPAAVGSL A. niger VYGDS -..-TCGQRVFYQFG

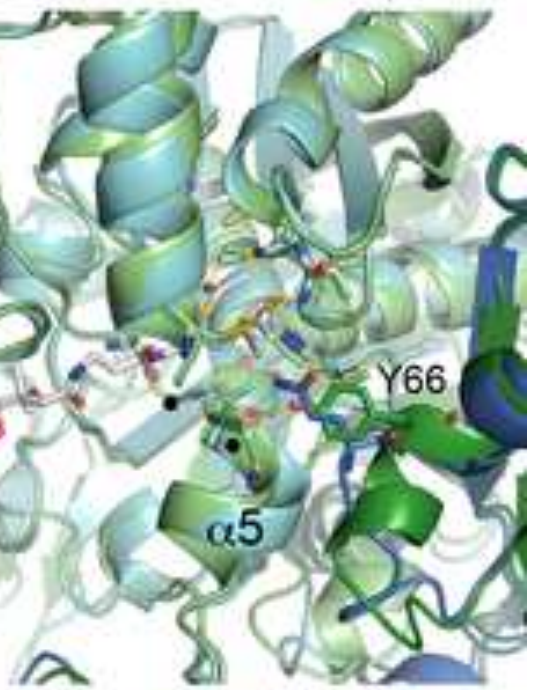

ReBktB ZrPhbA

$$
\alpha 5 \quad 150
$$

VDMMLG-ALHD VDTMIVDGLWD IDTMIKDGLTD IDSMIQDGLWD VDTMIVDGLWD VDTMIKDGLWC VDSMIKDGLSD ADSILCDGLTD BDLIVKDGLTD IDGVERDGLND -.-HPGLSRNV - - - HPGMGRTV ELLNPGLLQRY AVGNEVLKNDY - -AVKKFAQA - - KVETFAEA - - RLMEKEKA - - ELQKNRREA -..--QRQLDD MMAETRGITNA

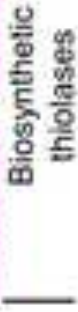

\section{\&}

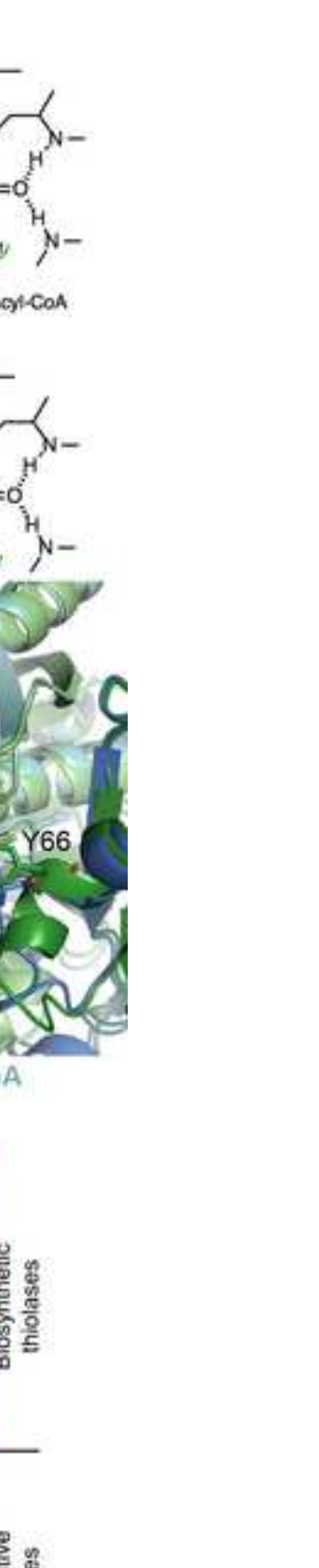




\section{a}
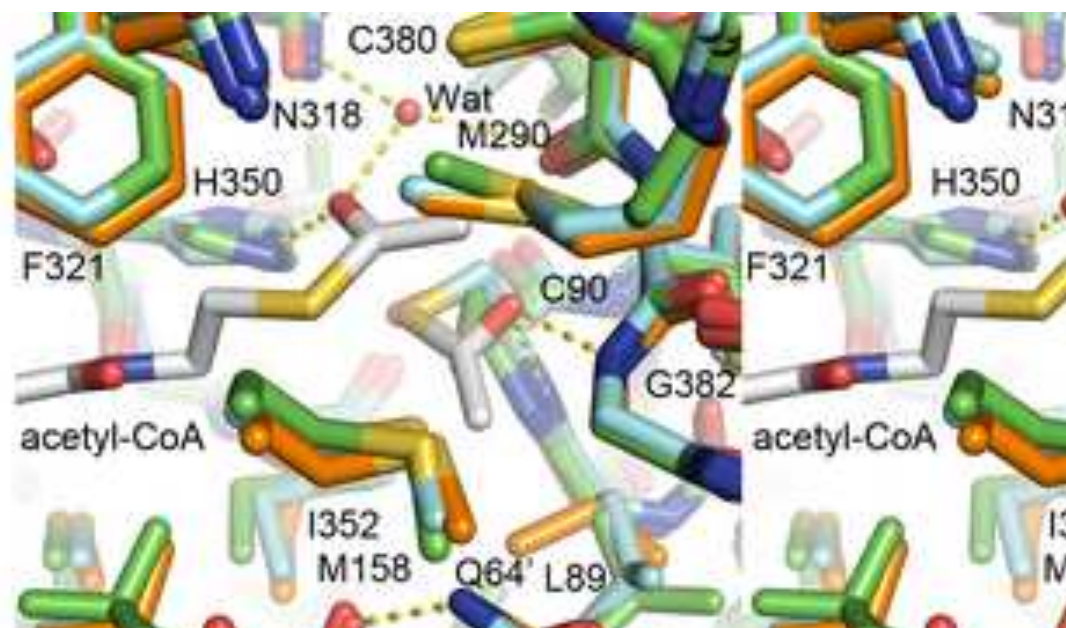

C380

N318 'eWat
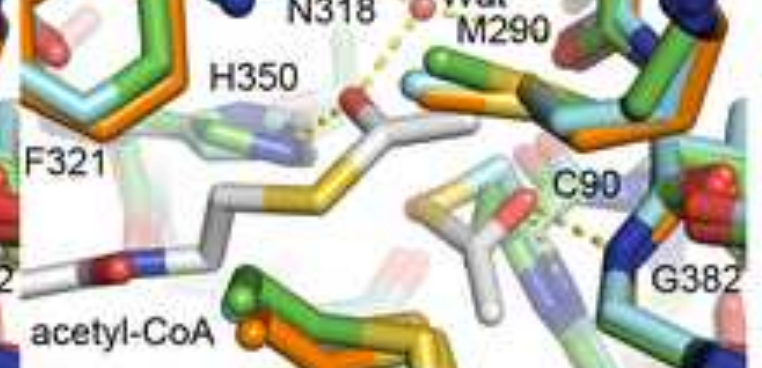

ZrPhbA

RePhbA

ReBktB
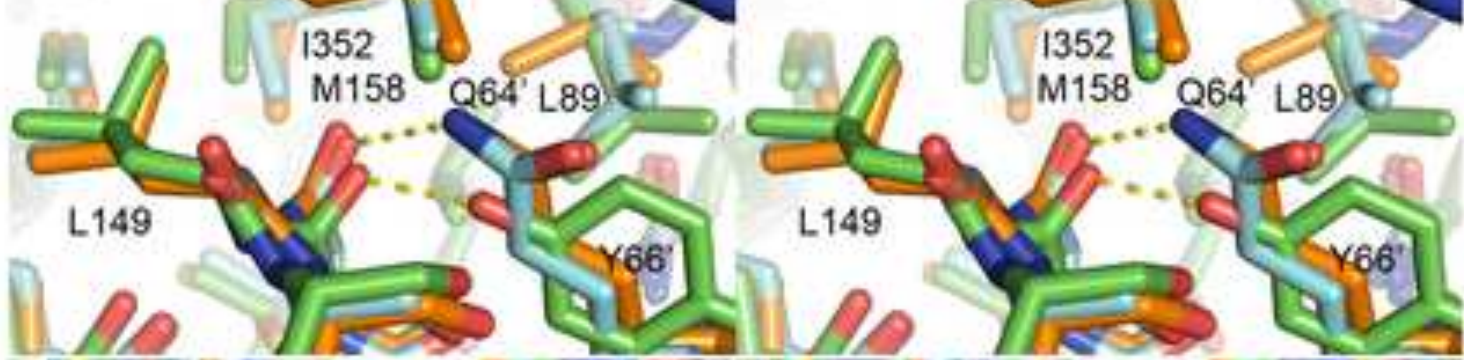

b
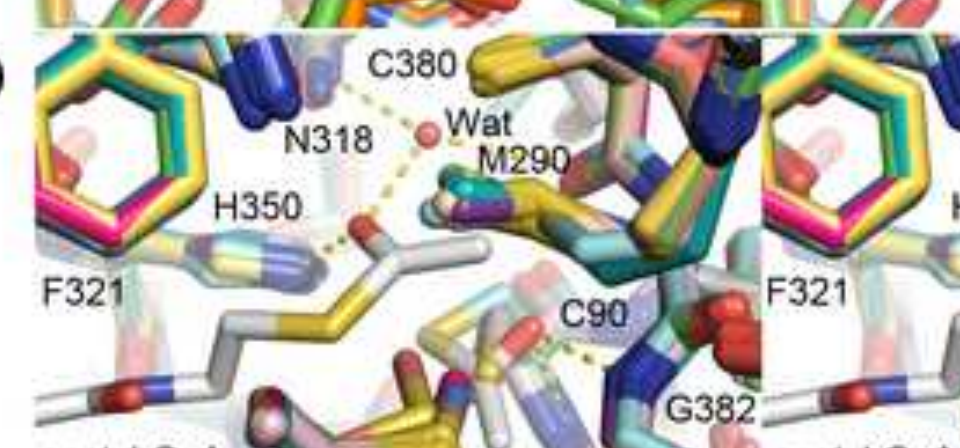

acetyl-CoA
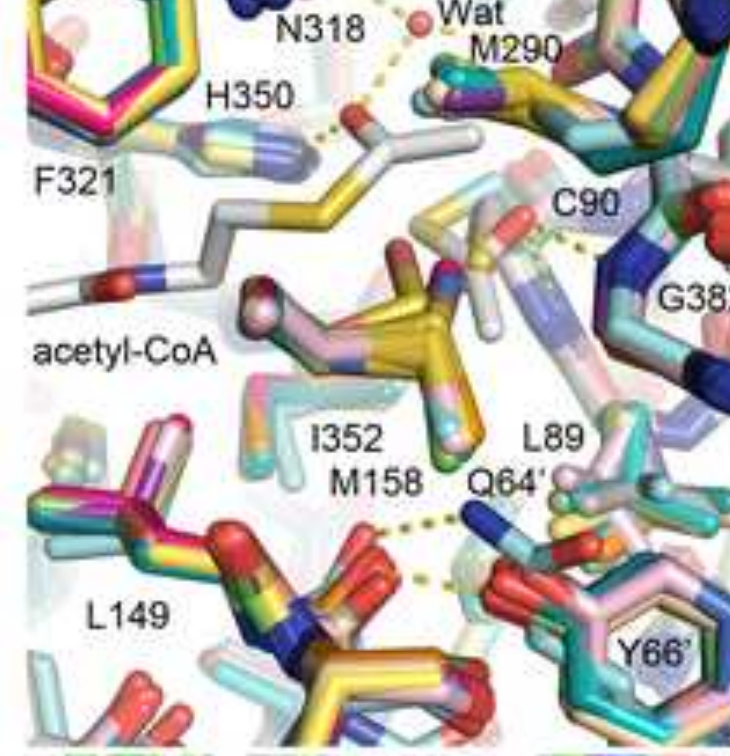

c
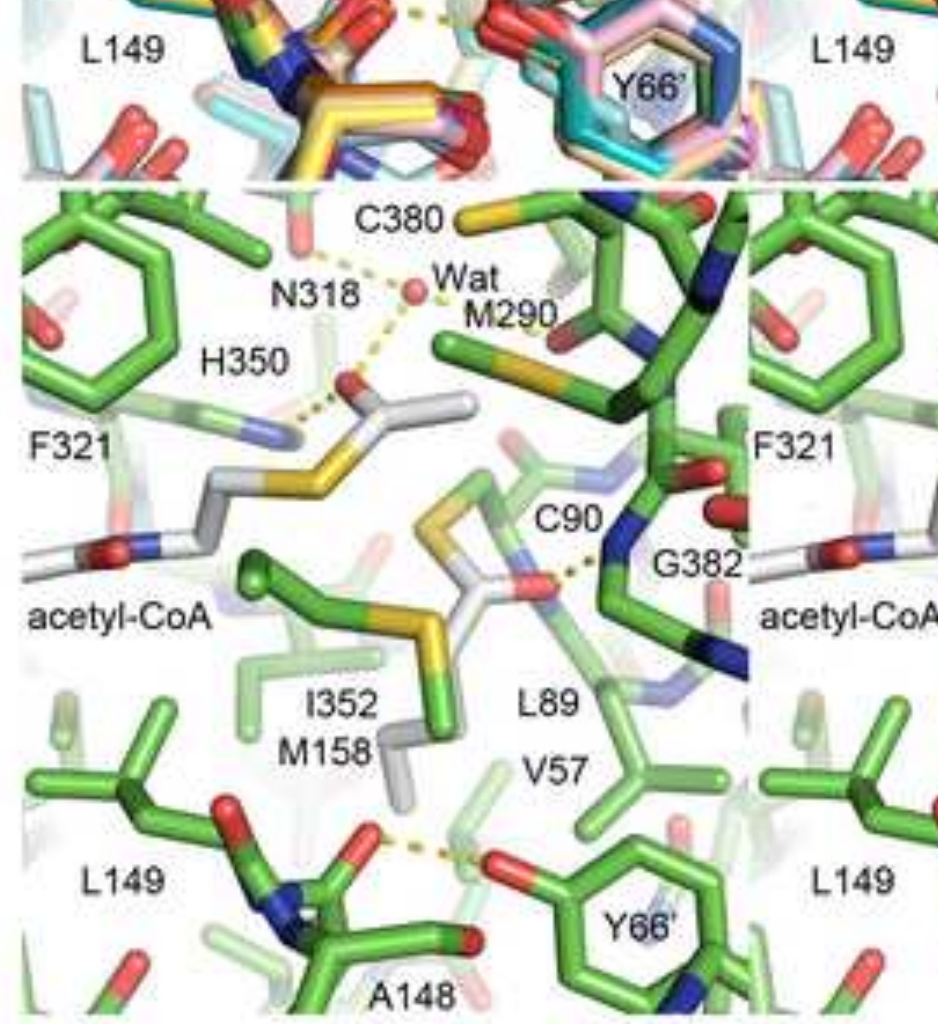

F321

H350

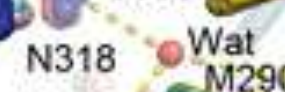

acetyl-CoA

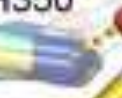

8

90

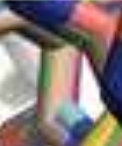

if

C90

ZrPhbA

ReBktB

(16 copies

from

unit cell)

\section{ReBktB}

acetyl-CoA and water from ZrPhbA structure, valeryl group modeled 

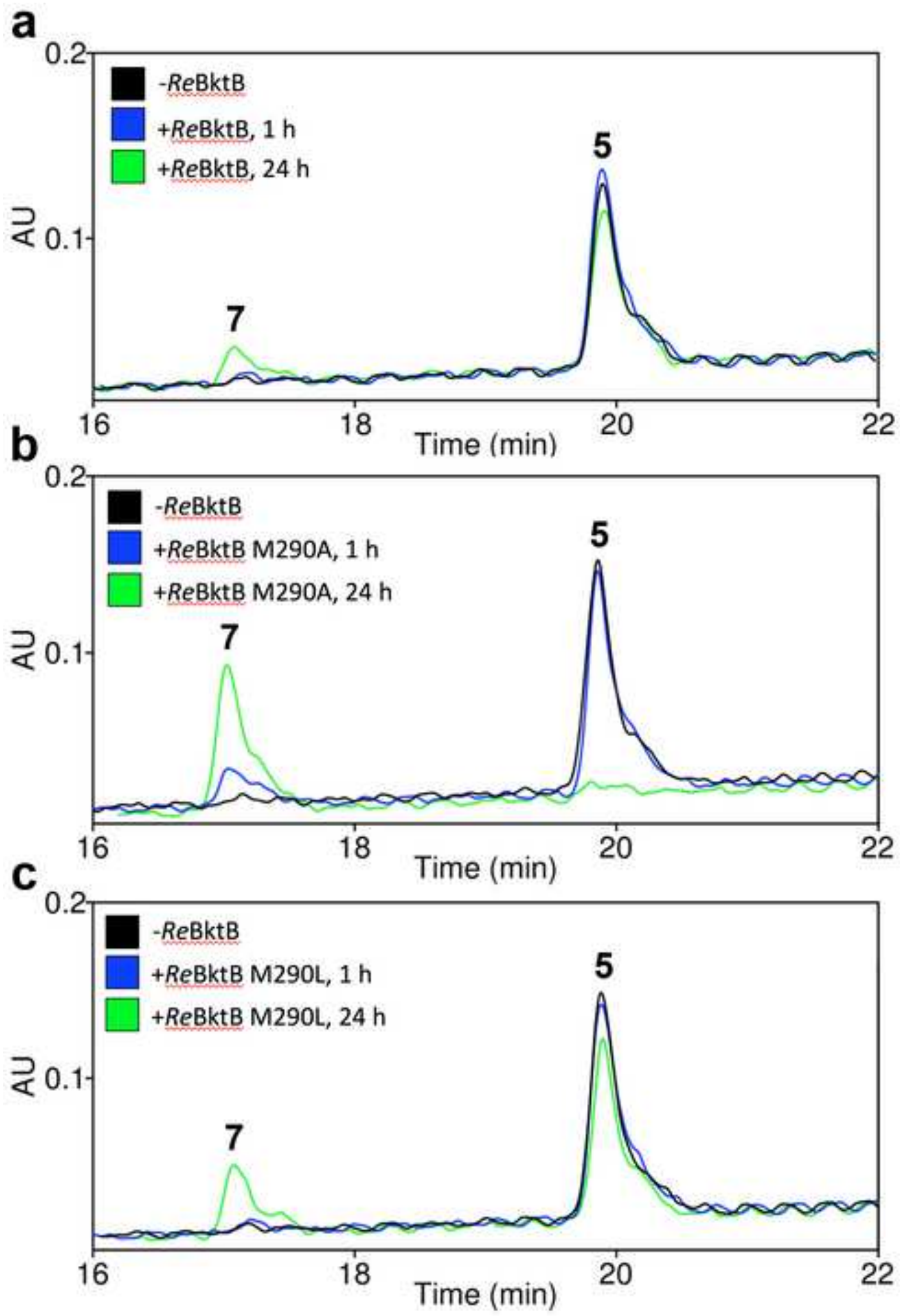
Table 1. Crystallographic data collection and refinement statistics for ReBktB

Data collection

Space group

Resolution $(\AA)$

Unit cell parameters

\begin{tabular}{|c|c|}
\hline$a, b, c(\AA)$ & $72.05,10$ \\
\hline$\alpha, \beta, \gamma\left(^{\circ}\right)$ & 89.97, \\
\hline$R_{\text {merge }}(\%)$ & $5.6(44$ \\
\hline Average $I / \sigma(I)$ & $10.4(2$. \\
\hline No. of observed reflections & 644,834 \\
\hline No. of unique reflections & 369,679 \\
\hline Redundancy & $1.7(1.7)$ \\
\hline \multicolumn{2}{|l|}{ Refinement } \\
\hline Resolution $(\AA)$ & $41.8-2$ \\
\hline Completeness (\%) & $92.9(7$ \\
\hline$R_{\text {work }} / R_{\text {free }}(\%)$ & $17.6 / 2$ \\
\hline \multicolumn{2}{|l|}{ No. of atoms } \\
\hline Overall & 46,064 \\
\hline Protein & 45,388 \\
\hline Water & 676 \\
\hline \multicolumn{2}{|l|}{ Average $B$-factors $\left(\AA^{2}\right)$} \\
\hline Overall & 42.2 \\
\hline Protein & 39.1 \\
\hline Water & 29.3 \\
\hline
\end{tabular}

Root-mean-square deviation
$P 1$

$50-2.00(2.03-2.00)$

$2.05,105.99,201.14$

$9.97,89.98,89.94$

5.6 (44.4)

$0.4(2.0)$

44,834

$69,679(18,535)$

.7 (1.7)

$1.8-2.01$ (2.06-2.01)

$2.9(78.7)$

$7.6 / 20.8(23.2 / 28.3)$ 


\begin{tabular}{cl}
\hline Bond lengths $(\AA)$ & 0.005 \\
Bond angles $\left(^{\circ}\right)$ & 0.68 \\
Ramachandran plot $(\%)$ & \\
Favored & 96.8 \\
Allowed & 2.2 \\
Outliers & 1.0 \\
PDB ID & $4 \mathrm{~W} 61$ \\
\hline Values for the highest-resolution shell are given in parentheses. \\
\hline
\end{tabular}

